\title{
Writing a thesis on a word processor
}

\author{
PETER MCDONALD
}

Advancement in hospital medicine in the major clinical specialties is almost impossible without the production of a carefully researched thesis. This task has never been easy, and as the competition for higher training posts becomes fiercer the urgency to submit a well presented $\mathrm{PhD}, \mathrm{MD}$, or $\mathrm{MS}$ thesis is very pressing for both the future consultant and the aspiring academic. I have recently completed a mastership thesis written without secretarial help on a microcomputer and I highly recommend this method.

The Rolls-Royce of word processors is the IBM Displaymaster $(£ 6300)$ but there are many small, cheaper business machines on the market and they all have their strengths and weaknesses. There are the BBC, the Commodore 64, the Apricot, the Peanut, the MTX, the Atari, the Sanyo microcomputer, the Mackintosh from Apple, the ingenious Sinclair QL, and the new range of portable word processors now flooding on to the market. The range of printers (dot matrix, top copy, etc) and interfaces is bewildering.

Storage systems and word processing programs may be included with the microcomputer. The reader has no need to go far for information. At my local newsagent last week I counted 40 different computer magazines on the shelf.

\section{Pros and cons}

The main advantage of a word processor is that, unlike even an experienced typist, it can never make new mistakes in the embedded text. Although moving around large sections or chapters is simple, errors may occur if this is not carried out carefully. Tables may be formulated as you want them to be. Nevertheless, the monitor and the 80 column facility of the microcomputer make the compilation of complex tables difficult. Teach yourself typing programs are available and learning to touch type is advisable, for keyboard skill will probably be a requirement for the properly trained doctor in the years to come.

The word processor may be used in the development of the thesis as well as for its final presentation. Protocols, data collection forms, consent forms, and other documents may be stored on disc. References can be stored after reading and recalled at a later date. A file program would be helpful for the latter and is not included in the basic word processing package.

\section{Principles}

The basic steps of word processing are simple and require no deep understanding of the way a microcomputer works. The procedure is as follows:

(1) Switching on; (2) loading word processor program on to

\footnotetext{
Wessex Regional Centre for Paediatric Surgery, Southampton General Hospital, Shirley, Southampton SO9 4XY

PETER MCDONALD, MS, FRCS, registrar
}

computer from floppy disc; (3) typing text; (4) editing text with edit control commands (for example, erasing, moving text, etc); (5) using special functions ("search and find," word counting, glossary, etc); (6) saving text in file on storage floppy disc; (7) locking files; (8) giving print formatting commands; (9) embedding print commands in file; (10) adjusting printer controls and inserting paper.

\section{My experience}

I used an Apple Ile microcomputer with a two disc operating system (DOS 3.3). One disc drive would have been adequate. I did not find the Applewriter very "user friendly." Although the manual is helpful, it is a daunting document.

As draft followed draft and the discs filled up, it was necessary that each draft (first, second, third, etc) should have its "filename" style so that it would be immediately recognisable. Hence the first draft became SECTION 1 (A), the second SECT 1A, the third S1A, and so on. Ideally each draft should be stored on a completely different set of discs.

A problem with the Apple Ile (and many other systems) is that it does not "save" files in the order of saving. The result is that files appear in random fashion on the screen when you give the "catalog" command and you cannot print out a record of file contents within the program. Discs had to be stored safely to avoid damage or proximity to magnetic fields. Actually the 5 inch $(13 \mathrm{~cm})$ floppy discs lasted well and I did not lose files from mechanical damage.

Checking that a file had been saved was a routine that I followed even when in a hurry to finish. After checking the file it must be "locked." If this is not done a new file with the same code will be saved in its place and the original erased. I learnt this the hard way. Text should be saved at hourly intervals in case of loss (mains failure, for instance). A back up floppy disc for duplication is advisable in case of loss or damage.

\section{PRINTING}

Printing instructions were quite complex and initially I made many errors. Nevertheless, the flexibility of printer commands was infinite. The text could be justified to the left, right, or centre; the paragraph margins altered; and the text paginated. Familiarity with these commands was of great importance for the final printout of the thesis.

Most universities have their own rules of layout and should be consulted at the outset. An 80 column line is too long for a $4 \mathrm{~cm}$ left margin and a $2 \mathrm{~cm}$ right margin on $A 4$. Several printouts were required to perfect the "formatting" instructions to the printer. These were reduced by displaying the page in a printed fashion on the computer before interfacing the printer itself. Print commands can also be embedded in the file.

I found that there were only a few functions (such as superscripts and subscripts) which were more difficult on the microcomputer than on a conventional typewriter.

Although I spent the best part of a year using the Applewriter program, there were plenty of functions that I used rarely - the word counting program, the find and replace function and glossary files, the case change function, the dictionary spelling file (an extra program), and so on. I acknowledge, however, that all of these functions might be useful. 


\section{$\cos \mathrm{T}$}

It is difficult to compare the cost of a word processor with that of a typist. I was lucky enough to have the free use of my department's computers and several discs. The overall cost of my thesis was low (artwork, paper, disposable carbon cartridges, photocopying, and binding). I would probably have needed three or four typewritten drafts (over $£ 400$ ). Instead, I had the luxury of seven or eight drafts from the microcomputer for much less.

More important, the thesis is truly my own creation and laid out exactly as I want it. It is a better product than it would have been. Moreover, producing future publications will be easy. The chapters of the thesis are embedded on those floppy discs which lie on my desk waiting for me to pitch them into "edit" mode.

\section{Basic rules}

Whichever microcomputer you (or your department) selects I suggest the following rules.

(1) Choose a simple word processing program-for example, Easywriter or Microsoft's Word.

(2) Choose a system with adequate memory. This does not have to be enormous. A RAM (random access memory) of $48 \mathrm{~K}$ (after the program has been loaded) is sufficient and will store about 20 double spaced pages on each file.

(3) A floppy disc operating system is preferable to tapes because of speed of access, although the new Microdrive system in the Sinclair QL may prove flexible and rapid enough for the average user.

(4) Your word processor will need occasional servicing and checking. This is especially true of disc drives, so a sensible warranty or guaranteed service arrangement is highly desirable.

(5) The choice of a good printer is very important, as it will be the product of this that will finally be taken to the university binders. A fast, good quality printer-for example, the Qumeis appropriate for departmental use with a dot matrix printer as a back up. If you want to produce your thesis at home then a cheaper, slow, top quality printer-for example, the Juki or the Epson-would be adequate. Make sure that the printer either has the typeface of your choice or has interchangeable daisy wheels available. A supply of Multi-strike (Single-strike is not necessary) carbon ribbon cartridges is needed, but they are expensive. Early drafts can be printed with cheaper and longerlasting cloth ribbons or on a dot matrix printer. Alternatively, a cheap dot matrix printer could be purchased for draft use and a daisy wheel printer hired for the final printout. I was quoted $£ 12$ an hour by one company.

(6) An 80 column card (now standard on most microcomputers used as word processors) should be obtained.

(7) Shop around. Although the "sales hype" for your custom has now reached fever pitch, many of the new, extended features of the word processing programs are unnecessary and expensive. The whole package should be between $£ 1000$ and $£ 2000$. Hiring a word processor is an expensive business, and rental at $10 \%$ of the purchase price a month is usual.

(8) As microcomputers may be used for many other purposes there seems little place for electronic typewriters or "dedicated" word processors. They have tiny memories and may be more expensive. Their only advantage is their simplicity. Two cheap dedicated word processors that might be worth considering, however, are the Brother CE-70 and Canon's AP 350.

(9) Get a keyboard that is the right size and one with solid but light to touch keys. The position of the alphabet is standard but the "control"," "reset," "escape," and "editing" keys may be awkwardly placed. Also the absence of characters (\$ in place of $£$, for instance) can be annoying. Some computers have added number keyboards like pushbutton telephone receivers, and these may be much easier to use than the conventional top row of a typewriter keyboard.

(10) A final word of warning. To date it is practically impossible to convert files written in one computer language to another. The disc operating system used-CP/M, PC-DOS, etc -and the size of the discs are important if compatibility with other users is being considered for the day when we will be passing our files routinely down the telephone via MODEMs.

I thank the department of surgery of the University of Southampton for the use of the computer facilities.
To what extent are the size of a car and the comfort of its seats a cause of sciatica?

The intradiscal pressure is maximal in the sitting position, and it is therefore essential that anyone who has been troubled by sciatica has a comfortable car seat. A well designed seat will support the lumbar spine so that the normal lumbar lordosis is maintained and should be long enough from front to back to provide adequate support for the thighs. In general, the larger cars, particularly those from Scandinavia, have well designed seats, whereas in smaller cars there is less space and height, making seating more cramped.-L KLENERMAN, consultant orthopaedic surgeon, London.

What toxic chemicals are used in glue and on what systems do they act? Which chemicals produce the pleasurable mental symptoms?

The deliberate inhalation of volatile hydrocarbons from a wide variety of solvent based products by children and teenagers presents a serious problem for those concerned with the care and welfare of the young. These products include adhesives containing toluene, acetone, and benzene; drycleaning substances containing trichlorethylene; dyes containing acetone and methylene chloride; nail polish removers containing acetone and amylacetate; petrol fumes containing benzene and naphthalene; and aerosols containing fluorocarbon. ${ }^{1-3}$ Nearly all volatile hydrocarbons are lipid soluble and are rapidly absorbed through the lungs. The blood brain barrier is rapidly penetrated by the lipophilic substances. Subjective effects vary from individual to individual and depend on the amount of solvent inhaled and the duration of inhalation. Toluene is the main solvent used in commer- cially available adhesives in Britain and is probably responsible for all the desired and toxic effects. ${ }^{12}$ Toluene is metabolised in hepatic microsomes by oxidation to benzoic acid; this is conjugated with glycine to form hippuric acid and is eliminated in this form through the kidneys. ${ }^{2}$ The physical sequelae of prolonged solvent use have been reported to include aplastic anaemia, acute hepatic and renal damage, cerebellar dysfunction, optic atrophy, encephalopathy, and peripheral neuropathy. ${ }^{3}$ Permanent neurological damage may also occur occasionally. ${ }^{2}$

Deaths after "glue sniffing" are usually due to accidents, asphyxiation, and inhalation of vomit. Nevertheless, the lack of morbidity and mortality in a group of 300 "glue sniffers" seen at a Glasgow clinic is reassuring. ${ }^{3}$ Toluene assay is a sensitive indicator of exposure to toluene. Blood concentration of toluene may be diphasic: an initial peak is followed by a trough reflecting lipid binding; the subsequent peak appears as toluene and is slowly released in the blood. ${ }^{2}$ The assay result may be positive up to several days after exposure to toluene. Benzene has a well recognised myelotoxic potential. Neuropathy has been attributed to $\mathrm{N}$-hexane inhalation. Solvent vapours, and particularly ketones, may produce irritation of the eyes, nose, throat, and upper respiratory tract. Fluorocarbons sensitise the heart to epinephrine induced arrhythmias. ${ }^{3}$ Severe cardiac arrhythmias, intensified by hypercapnia, stress, or activity are conr idered to be the most likely explanation for the sudden death after aerosol inhalation.-I souRINDHRIN, senior registrar, Glasgow.

'Oliver JS, Watson JM. Abuse of solvents "for kicks" -a review of 50 cases. Lancet 1977; i:84-7. King MD, Day RE, Oliver JS, Lush M, Watson JM. Solvent encephalopathy. Br
Med F 1981;283:663-5. Sourindhrin I, Baird JA. Management of solvent misuse: a Glasgow community
approach. Br $₹$ Addict (in press). 